\title{
OPTIMAL HMM PROCESSING OF DIFFERENTIALLY PHASE MODULATED COMMUNICATION SYSTEMS
}

\author{
Emily Lane, Jason Ford and John Moore \\ Depart. of Systems Engineering \& CRASys, RSISE, ANU, Canberra ACT Australia 0200. \\ Contact Jason on Ph +61(6)2494581 or email Jason.Ford@anu.edu.au.
}

\begin{abstract}
This paper investigates demodulation of differentially phase modulated signals (DPMS) using optimal HMM filters. The optimal HMM filter presented in the paper is computationally of order $N^{3}$ per time instant, where $N$ is the number of message symbols. Previously, optimal HMM filters have been of computational order $N^{4}$ per time instant. Also, suboptimal HMM filters have be proposed of computation order $N^{2}$ per time instant. The approach presented in this paper uses two coupled HMM filters and exploits knowledge of their interdependence to achieve computational gains.

A simulation study is also presented.
\end{abstract}

\section{INTRODUCTION}

The capacity of wireless digital communication system is often limited by fading in the transmission channel. One common approaches to alleviate this difficulty is through the use of decision feedback equalisation (DFE). For example, a DFE approach was used in [2], via a coupled Kalman filter (KF) and hidden Markov model (HMM) filter, to demodulate quadrature amplitude modulated (QAM) signals. At each time instant the HMM filter estimates the message sent and then the KF uses this estimate to update its estimate of the channel. The disadvantage of this approach arises if errors in the estimation of the message sequence occur. Errors in the estimate for the message signal cause the channel estimate to diverge from the true channel; the whole DFE structure then breaks down.

A second common approach to alleviate difficulties resulting from channel fading is though the use of differential phase modulated signals (DPMS). The major advantage of difference signalling is that message information is encoded in the differences between successive transmitted signals. For this reason tracking of the

The authors wish to acknowledge the funding of the activities of the Cooperative Research Centre for Robust and Adaptive Systems by the Australian Government under the Cooperative Research Centres Program. channel is not required and as long as the channel is slowly time-varying then its effect on the symbols can be largely ignored.

To formulate DPMS into a HMM structure, two finite dimensional indicator vectors are used. One to represent differential message signal being sent at each time instant and the other to represent actual message symbol being sent at each time instant. In previous papers optimal HMM filters have been developed which consider the whole state space spanned by the modulation system and these schemes are computationally of order $N^{4}$ per time instant, where $N$ is the number of message symbols. Another approach developed is to use a suboptimal solution which uses coupled conditional HMM filters which assume independence between the two indicator vectors, where there is obviously dependence [4]. This sub-optimal scheme is computationally of order $N^{2}$.

The key contribution of this paper is to use coupled conditional HMM filters, in the same vein as the suboptimal approach, which exploit the interdependence between the sent message and the differential message to ensure optimality of the filters.

This paper is organised as follows: In Section 2, we formulate the HMM, signal model and channel model for a DPMS system. In Section 3, we introduce our optimal HMM filter. In Section 4, we present a coupled filter formulation for the optimal HMM filter. In Section 5, a simulation study is presented. Finally, some conclusions are presented in Section 6.

\section{PROBLEM FORMULATION}

In this section we introduce the HMM signal model in state form and the channel model.

\subsection{Signal Model}

Two HMM indicator functions are used to represent the state of the differential modulation scheme. Assume the signals from the message source, $f_{k}$, space belongs 
to a finite-discrete set. That is, let $f_{k}$ be a real valued discrete-state discrete time process. Without loss of generality, $f_{k}$ can be thought to belong to the message space $\{1,2,3, \ldots, N\}$, where $N$ is the number of possible message symbols.

Assumption on the message source

$f_{k}$ is a first order Markov process

Then the message can be represented by a sequences of Markov indicator functions, $X_{k}^{f} \in\left\{e_{1}, \ldots, e_{n}\right\}$ where $e_{i}=(0, \ldots, 0,1,0, \ldots 0)^{\prime}$ with 1 in the $i$ th position.

The transition probability matrix of the Markov process is

$$
\mathbf{A}=\left(a_{i j}\right) \quad \leq i, j \leq N
$$

where

$$
a_{i j}=P\left(X_{k+1}^{f}=e_{i} \mid X_{k}^{f}=e_{j}\right)
$$

so that

$$
E\left[X_{k+1}^{f} \mid X_{k}^{f}\right]=A X_{k}^{f}
$$

where $E[$.$] denotes the expectation operator. We also$ denote $\left\{\mathcal{F}_{l}, l \in \mathcal{Z}^{+}\right\}$the complete filtration generated by $X^{f}$, that is, for any $k \in \mathcal{Z}^{+}, \mathcal{F}_{k}$ is the complete filtration generated by $X_{k}^{F}, l \leq k$.

Lemma 1 The dynamics of $X_{k}^{f}$ are given by the state equation

$$
X_{k+1}^{f}=A X_{k}^{f}+M_{k+1}
$$

where $M_{k+1}$ is a $\left(A, \mathcal{F}_{k}\right)$ martingale increment, in that $E\left[M_{k+1} \mid \mathcal{F}_{k}\right]=0$.

The symbol transmitted in a differential modulation scheme is the modulo sum of the message sequence. If we let $\theta_{k}$ denote the symbol sent at time $k$ and $S_{k}=\left(\sum_{i=1}^{k} I_{i}\right) \bmod N$, denote the modula sum of the message sequence, then

$$
\theta_{k}=Z_{f}^{\left(S_{k}\right)}
$$

where $Z_{f}=\left\{Z_{f}^{(1)}<\ldots, Z_{f}^{(N)}\right\}$ are complex number that denote the signal constellation using in a QAM or similar transmission scheme.

We also represent the symbol sent by the indication function, $X_{k}^{\theta}$ where $X_{k}^{\theta}=e_{S_{k}} \in\left\{e_{1}, \ldots, e_{N}\right\}$.

Lemma 2 The indicator function $X_{k}^{\theta}$ is given from $X_{k}^{f}$ as follows

$$
\begin{aligned}
X_{k+1}^{\theta} & =D\left(X_{k+1}^{f}\right) X_{k}^{\theta} \text { or } \\
X_{k+1}^{\theta} & =f\left(X_{k}^{\theta}\right) X_{k+1}^{f}
\end{aligned}
$$

where $D\left(e_{i}\right)=S^{n^{\prime}} e_{i}$ the shift operator. Note, $S$ is defined as

$$
S=\left(\begin{array}{ccccc}
0 & 0 & \ldots & 0 & 1 \\
1 & 0 & \ldots & 0 & 0 \\
0 & 1 & \ldots & 0 & 0 \\
\vdots & \vdots & \ddots & \vdots & \vdots \\
0 & 0 & \ldots & 1 & 0
\end{array}\right)
$$

and $\underline{n}=(1, \ldots, N)^{\prime}$.

Proof Proof of (2.7) comes from the definition of DPMS. Proof of (2.8) comes from noting that $X_{k+1}^{f}$ and $X_{k}^{\theta}$ are indicator functions and hence non-linear functions are simply linear functions, see [3].

\subsection{Channel Model}

The signal $\theta_{k}$ is transmitted via a channel which can cause amplitude attenuation and phase shift. The channel can be represented as a multiplicative disturbance, $c_{k}$.

The observation process $y_{k}$ is thus assumed to have the form

$$
y_{k}=c_{k} m_{k}+w_{k}
$$

where $m_{k}=Z_{f} X_{k}^{\theta}$. We also define $Y_{k} \triangleq\left(y_{o}, \ldots, y_{k}\right)$. We assume $w_{k}$ is complex with real and imaginary parts that are i.i.d., with zero mean and Gaussian and $\mathcal{Y}_{l}$ is the complete filtration generated by $y_{k}, k \leq l$. As a consequence,

$$
E\left[w_{k+1} \mid \mathcal{F}_{k} \vee \mathcal{Y}_{k}\right]=0
$$

In cartesian coordinates, with the output written as

$$
Y_{k}=\left(\begin{array}{c}
y_{k}^{R} \\
y_{k}^{I}
\end{array}\right)
$$

we obtain

$$
\begin{aligned}
\left(\begin{array}{c}
y_{k}^{R} \\
y_{k}^{I}
\end{array}\right)= & \left(\begin{array}{cc}
m_{k}^{R} & -m_{k}^{I} \\
m_{k}^{I} & m_{k}^{R}
\end{array}\right)\left(\begin{array}{c}
c_{k}^{R} \\
c_{k}^{I}
\end{array}\right)+\left(\begin{array}{c}
w_{k}^{R} \\
w_{k}^{I}
\end{array}\right) \\
= & \left(\begin{array}{cc}
\left(Z_{f}^{R}\right)^{\prime} X_{k}^{\theta} & -\left(Z_{f}^{I}\right)^{\prime} X_{k}^{\theta} \\
\left(Z_{f}^{I}\right)^{\prime} X_{k}^{\theta} & \left(Z_{f}^{R}\right)^{\prime} X_{k}^{\theta}
\end{array}\right)\left(\begin{array}{c}
c_{k}^{R} \\
c_{k}^{I}
\end{array}\right) \\
& +\left(\begin{array}{c}
w_{k}^{R} \\
w_{k}^{I}
\end{array}\right)
\end{aligned}
$$

where the notation $Z_{f}^{R}$ and $Z_{f}^{I}$ means the real and imaginary part of $Z_{f}$ respectfully, and has the same interpretation for the other signals. 


\section{OPTIMAL HMM REPRESENTATION}

In this section we introduce a new formulation to the problem of finding the optimal HMM filter for DPMSs. Previously, the optimal HMM filter was thought to require order $N^{4}$ calculations per time instant. The filter was constructed as follows.

Let $\mathcal{X}_{k}^{0}$ denote the space of the modulation scheme. This space is also represented by the indicator functions $X_{k}^{f}$ and $X_{k}^{\theta}$. The approach taken in the previous formulation of the optimal HMM filter is obtain $\mathcal{X}_{k}^{0}$ from the Kronecker product of these indicator functions. That is,

$$
\mathcal{X}_{k}^{0}=X_{k}^{f} \otimes X_{k}^{\theta}
$$

where $\otimes$ is the Kronecker product. $\mathcal{X}_{k}^{0}$ is known to be a Markov process and standard HMM filtering theory can be applied. However, $\mathcal{X}_{k}^{0}$ is $\left(N^{2} \times 1\right)$ and hence the filter calculations are of order $N^{4}$.

If instead denote the modulation scheme space by $\mathcal{X}_{k}$ and formed as follows

$$
\mathcal{X}_{k}=X_{k}^{f} X_{k}^{\theta^{\prime}}
$$

then we note that

$$
\begin{aligned}
X_{k}^{f} & =\mathcal{X}_{k} \underline{1}_{N} \text { and } \\
X_{k}^{\theta} & =\mathcal{X}_{k}^{\prime} \underline{1}_{N}
\end{aligned}
$$

where $\underline{1}_{N}=(1, \ldots, 1)^{\prime}$, an $N$-vector of ones.

\section{Remarks}

1. $\mathcal{X}_{k}$ is a $(N \times N)$ matrix while $\mathcal{X}_{k}^{0}$ is a $\left(N^{2} \times 1\right)$ vector.

2. For vectors, the Kronecker product and outer product have the same terms, they are simply stored differently.

3. While the difference in definition of $\mathcal{X}_{k}$ and $\mathcal{X}_{k}^{0}$ is not great, $\mathcal{X}_{k}$ does highlight the structure in the problem caused by the nature of the modulation scheme.

Lemma 3 The dynamics of $\mathcal{X}_{k}$ are given by the state equation

$$
\mathcal{X}_{k+1}=\sum_{i=1}^{N} e_{i}\left(\mathcal{A}_{i}(A) \mathcal{X}_{k}(i,:)^{\prime}\right)^{\prime}+\mathcal{M}_{k+1}
$$

where $\mathcal{X}_{k}(i,:)$ represents the $i$ th row of matrix $\mathcal{X}_{k}, \mathcal{M}_{k+1}=$ $M_{k+2} e_{i+j}^{\prime}$ and $\mathcal{A}_{i}(A)$ is some vector related to $A$.
Proof Express $\mathcal{X}_{k+1}$ as $X_{k+2}^{f} X_{k+1}^{\theta^{\prime}}$. Substitute in (2.7) and (2.5) and from noting that $\mathcal{X}$ in a zero matrix except in one element the result follows.

Since from Lemma 3 it is clear that the dynamic of $\mathcal{X}_{k}$ can be considered as $N$ parallel independent HMMs we use standard HMM filter techniques on each of the rows of $\mathcal{X}$ to obtain an estimate of $\hat{\mathcal{X}}_{k}=E\left[\mathcal{X}_{k} \mid Y_{k}\right]$. That is,

$$
\hat{\mathcal{X}}_{k+1}(i,:)^{\prime}=N_{k} \mathbf{B}_{k+1} \mathcal{A}_{i} \hat{\mathcal{X}}_{k}(i,:)^{\prime}
$$

where $N_{k}$ is a normalising factor for each row, $\mathbf{B}_{k}=$ $\operatorname{diag}\left(b_{k}\left(e_{1}\right), \ldots, b_{k}\left(e_{N}\right)\right.$, and $b_{k}\left(e_{i}\right)=p\left[Y_{k} \mid X_{k}^{\theta}=e_{i}\right]$.

From $\hat{\mathcal{X}}_{k+1}$ estimates for two indicator function can be found using property (3.3).

\section{Remarks}

1. Lemma 3 simply shown that $\mathcal{X}_{k}$ evolves like $N$ parallel HMMs. Each row represent a separate Markov chain.

2. This structure is hidden in the $\mathcal{X}_{k}^{O}$ formulation.

3. This filter required of the order $N^{3}$ calculations per time instant.

\section{COUPLED FILTERS FORMULATION}

In this section we present a more convenient formulation of our optimal HMM filter using couple conditional HMM filters. In this formulation we do not directly create $\mathcal{X}_{k}$ but rather the conditional HMM filters of $X_{k+1}^{f}$ and $X_{k+1}^{\theta}$, exploiting the interdependence between the signals.

Let $\hat{X}_{k+1}^{f}$ and $\hat{X}_{k+1}^{\theta}$ denote the conditional filtered normalised state estimates of $X_{k+1}^{f}$ and $X_{k+1}^{\theta}$ respectively. That is, by definition

$$
\begin{aligned}
\hat{X}_{k+1}^{f} & =E\left[X_{k+1}^{f} \mid Y_{k}\right] \\
\hat{X}_{k+1}^{\theta} & =E\left[X_{k+1}^{\theta} \mid Y_{k}\right]
\end{aligned}
$$

Firstly, consider the intermediate conditional state estimate, $\hat{X}_{k+1 \mid i}^{f}$, given by

$$
\hat{X}_{k+1 \mid i}^{f}=E\left[X_{k+1}^{f} \mid Y_{k}, X_{k}^{\theta}=e_{i}\right]
$$

From Bayes rule it is clear that

$$
\hat{X}_{k+1}^{f}=\sum_{i=1}^{N} \hat{X}_{k+1 \mid i}^{f} \hat{X}_{k}^{\theta}(i)
$$

where $\hat{X}_{k}^{\theta}(i)$ is the ith element if $\hat{X}_{k}^{\theta}$. Note that $\hat{X}_{k}^{\theta}(i)=$ $P\left(X_{k}^{\theta}=e_{i} \mid Y_{k}\right)$. 
Lemma 4 The following forward recursion exists to estimate $\hat{X}_{k+1 \mid i}^{f}$

$$
\hat{X}_{k+1 \mid i}^{f}=N_{k}^{(1)} B_{k+1 \mid i} A \hat{X}_{k}^{f}
$$

where $B_{k \mid i}=\operatorname{diag}\left(b_{k \mid i}\left(e_{1}\right), \ldots, b_{k \mid i}\left(e_{N}\right)\right)$ with $b_{k \mid i}\left(e_{j}\right)=$ $P\left[y_{k} \mid X_{k}^{\theta}=e_{i}, X_{k+1}^{f}=e_{j}\right]$ and where $N_{k}^{(1)}$ is a normalising factor.

Proof Follows from assumption (2.1).

Lemma 5 The conditional filtered normalised state estimates $\hat{X}_{k+1}^{\theta}$ is given by

$$
\hat{X}_{k+1}^{\theta}=N_{k}^{(2)} \mathcal{B}_{k+1} \sum_{i=1}^{N} f\left(e_{i}\right) \hat{X}_{k+1 \mid i}^{f}
$$

where $\mathcal{B}_{k \mid i}=\operatorname{diag}\left(b_{k}\left(e_{1}\right), \ldots, b_{k}\left(e_{N}\right)\right)$ with

$b_{k}\left(e_{i}\right)=P\left[y_{k} \mid X_{k}^{\theta}=e_{i}\right]$ and $N_{k}^{(2)}$ is a normalising factor.

Proof Follows from (2.8)

Lemma 6 The conditional filtered normalised state estimates $\hat{X}_{k+1}^{f}$ is given by

$$
\hat{X}_{k+1}^{f}=N_{k}^{(3)} \sum_{i=1}^{N} \hat{X}_{k+1 \mid i}^{f} e_{i}^{\prime} \hat{X}_{k+1}^{\theta}
$$

where $N_{k}^{(3)}$ is a normalising factor.

Proof Follows from definition of $\hat{X}_{k+1}^{f}$

Application of these last three lemmas gives a recursive filter for estimating $\hat{X}_{k+1}^{f}$, the signal of interest, at each time instant.

\section{Remarks}

1. This filter generates the same results as the filter given in the previous section.

2. The primary difference between the sub-optimal approach in [4] and here appears in Lemma 5. The suboptimal approach would update as follows

$$
\hat{X}_{k+1}^{\theta}=\mathcal{B}_{k+1} \hat{X}_{k}^{\theta}
$$

3. These filters take $O\left(N^{3}\right)$ calculations per time step to implement, compared with $O\left(N^{4}\right)$ for previous optimal filters and $O\left(N^{2}\right)$ for previous suboptimal filters.

\section{SIMULATIONS}

To evaluate the performance of our DPMS HMM filter, we compared it with the performance of the DFE scheme implemented in [2]. It should be noted that the DFE scheme requires $O\left(N^{2}\right)$ calculations per time instant and our HMM scheme requires $O\left(N^{3}\right)$ calculations per time instant. Two modulated signals where generated from the same binary message source A QAM signal was generated to test the DFE and a DPSK signal to test our differential HMM filter. Both signals were subjected to that same channel interference, a slowly fading white additive noise channel. The schemes were compared over a range of signal to noise ratios.

In lower signal to noise ratios, our HMM filter performed better. The primary reason for this begin that the DFE does not regain tracking after errors, while the DPMS scheme does. In particular, when the channel faded to a null, both schemes made errors, however, the DFE did not regain tracking after the null.

\section{CONCLUSION}

In this paper we have presented a optimal HMM filter for demodulation of differentially encoded signals. This optimal filter is requires $\mathrm{O}\left(N^{3}\right)$ calcualtions per time step, where $N$ is the number of message symbols, compared to previous optimal schemes which were computationally of order $N^{4}$.

The optimal HMM filter presented in this paper exploits more of the structure in the demodulation problem then previous optimal HMM filters do. A simulation example was given.

\section{REFERENCES}

[1] R. J. Elliott, L. Aggoun and J. B. Moore, Hidden Markov Models, Estimation and Control, Springer-Verlag, 1995.

[2] I.B. Collings and J.B. Moore, Adaptive HMM Filters for Signals in Noisy Fading Channels, Proc. of Int. Conf. on Acoustics, Speech \& Signal Processing ICASSP94, Adelaide, Australia, Vol. 3, pp 305-308, 1994

[3] J.J. Ford and J.B. Moore, On Adaptive HMM state estimation, submitted to IEEE Trans. on Signal Processing, Oct. 1995.

[4] I.B. Collings, Hidden Markov Model Signal Processing and Control, PhD thesis, Australian National University, January 1995. 